\title{
Pollination strategies in the face of pollinator decline Pierre-Olivier Cheptou
}

\author{
CEFE UMR 5175, CNRS, Université de Montpellier, Université Paul-Valery \\ Montpellier, EPHE - 1919 route de Mende, 34293 Montpellier cedex 05, France \\ Phone: +33 (4) 67613307 \\ Email: pierre-olivier.cheptou@cefe.cnrs.fr
}

\begin{abstract}
The recent pollinator decline in developed countries has been documented in many parts of the world and its anthropogenic causes are now identified. Because pollinators contribute to the sexual reproduction of many plant species, pollinator decline constitutes a potential threat on insect-pollinated plant populations. Moreover, it is susceptible to impede pollination services to crops in agriculture. In this paper, I review the empirical evidence that pollinator decline translates (or not) into pollen limitation for plants and alters plant-pollinator networks. I also analyse the possibility of short-term evolution of plant pollination strategies. In particular, I discuss how changes in pollinator fauna constitutes new selection pressures for plants and how plant reproductive traits are able to respond rapidly to such new selection regime. Mating system evolutionary theory thus predicts that short-term evolution may allow plant populations to adapt to pollinator decline, potentially rescuing populations, as predicted by the evolutionary rescue theory. Nevertheless, mating system theory and empirical data support the idea that evolutionary rescue through mating system adaptation is not always possible. Finally, I discuss how plant evolution may disturb plant-pollinator interactions potentially breaking major trophic links in ecosystems, which could in turn reinforce pollinator's extinction in the future.
\end{abstract}

Keywords: Pollinator decline, Plant pollination strategies, Adaptation, Evolutionary rescue, Plant-pollinator network. 


\section{Introduction}

A recent study reported that more than $75 \%$ of insects declined during the last thirty years (Hallmann et al. 2017). Among them, pollinator decline has become a feature of particular interest because of their well-identified role in ecosystems: they allow sexual reproduction of many plant species. Moreover, by pollinating crops, pollinators have been identified as a major ecosystemic service in agriculture. About $85 \%$ of crops rely at various degrees on insect pollination, which concerns about $40 \%$ of crop production.

Pollinator decline is sometimes assimilated to bee decline called the "Colony collapse disorder" (Goulson et al. 2015). This is however not the unique group of pollinator declining. Figure 1 illustrates the contraction of Bombus distiguendus in the United Kingdom. According to the National Biodiversity Network data (UK), the species was recorded in the whole United Kingdom and Ireland in the past. Since 2000, the species is restricted to the northern parts of Scotland. Changes in land use (e.g. loss of flowering grasslands, intensive agriculture,...), parasites and pesticides have been identified as important causes of decline in developed countries (Goulson et al. 2015). Because plant depends on pollinators to produce their seeds and thus to maintain a sustainable demography, pollinator decline has the potential to disturb plant reproduction and to induce plant population extinction, with cascading effects on other species linked by trophic relationships. Furthermore, experiments have shown that it may alter the pollination of crops and reduce crop yield (Perrot al. 2018).

In the 1980's, evolutionary ecologists and population geneticists have developed an important theoretical framework analyzing plant mating system evolution. Coupled with a large corpus of empirical studies, the major selective factors have been identified and in particular, the role of pollination environment. While mating system evolution models have been mainly used as a way to interpret patterns in natural populations, the pollinator's crisis has stimulated studies analyzing microevolution on mating systems. To date, empirical studies are scarce but several experimental studies have shown convincingly that floral traits and mating system traits can evolve rapidly (Roels and Kelly 2011; Gervasi and Schiestl 2017).

This paper aims at exploring the impact of pollinator decline on entomophilous plants. More specifically, I discuss (1) how pollinator decline is susceptible of modifying plant communities (2) how pollinator decline translates into pollen limitation for plant populations 
and (3) the potential for plants to adapt to pollinator decline and its consequences on population demography and trophic relationships in ecosystems.

\section{1-Pollinator decline and plant communities}

Plant pollinator interactions are in most cases mutualistic, the plants provide food for the pollinators (nectar and pollen), while the pollinators allow sexual reproduction through pollen transport. According to the Intergovernmental Science Policy Platform on Biodiversity and Ecosystem Services (IPBES), more than 300000 plant species benefit from insects for their reproduction i.e. $87.5 \%$ of the total flora (Rhodes 2018). We can thus make the prediction that changes in pollinator composition should affect plant community. Curiously, such interactions have traditionally been little studied in community ecology (E-Vojtkó et al. 2020). Community ecology has rather focused on functional traits (morphology, physiology, phenology) with little interest on reproductive traits and pollination. Recent empirical studies have however demonstrated that reproductive traits are linked to community structure. Using a large dataset of more than 600000 trees and historical information documenting recently colonized areas, Rejou-Mechain and Cheptou (2015) showed that sexual system (dioecy) was the best predictor of young successional communities in Central Africa. Importantly, functional traits classically associated with young successional areas (e.g. N-fixing) were not found to be associated with dioecy. This thus suggests that dioecy does not influence plant community composition through trait association (at least those measured) but that dioecy, on its own, advantageous in young successional stages.

In 2006, Biesmeijer et al. (2006) published an important paper about the mutual influence of pollinators and plants in the context of pollinator decline. In their study, the authors compared plant composition and bee and hoverfly assemblages in Britain and the Netherlands several decades apart (pre- versus post-1980). The authors showed a parallel decline of insect pollinated plants and pollinators, thus demonstrating that plant and pollinators composition are interdependent in ecosystems. While it is tempting to conclude on the causal role of pollinator decline (caused by external factors) on plant species composition, the authors rigorously conclude that their data do not allow to conclude whether the decline of plants precede the loss of associated pollinators or the reverse. Using historical surveys in Britain, Baude et al (2016) have shown that nectar resources have greatly reduced between the 1930s and 1970s. These trends are consistent with pollinator declines but, again, does not allow to 
determine the causal connection of the parallel decline. The difficulty of attributing a causal connection in such a parallel decline relies on the structure of plant pollinator networks and more specifically whether pollinators or plants are limiting in ecosystems. Since 2000's, mutualistic networks have been subject to intensive interests in theoretical community ecology and synthetic metrics have been defined to characterize networks (Bascompte et al. 2003; Vazquez et al. 2009). While network structures can vary according to ecosystemic specificities, the general feature is the imbalanced number of pollinator species and plant species: there are approximately four times more pollinator species than plant species. The interactions are asymmetric, which means that specialist plant species tend to interact with generalist pollinator species. Astegiano et al. (2015) studied the robustness of networks to pollinator loss. Studying 13 plant-pollinator networks from various parts of the world, they showed that networks are globally robust to moderate pollinator loss. Such community ecology studies highlight the fact that plant populations may be resilient to pollinator decline and that it will not necessarily generate pollen limitation for plants.

\section{2-Pollinator decline and pollen limitation in natural populations}

At the population level, pollination ecology has for a long time revealed that most of plant populations are pollen-limited for seed production in natural conditions. In a survey of more than 250 plant species, Burd (1994) showed that more than 150 were significantly pollenlimited. Leaving ovules unfertilized may appear at first sight as non-adaptive since producing more seeds, by self-fertilizing the remaining ovules would a priori increase individual fitness. Pollen limitation is classically estimated by experiments comparing seed (or fruit) production under natural pollination and when plants are pollen supplemented. More refined techniques have been developed to estimate reproductive assurance by manipulating the possibility of selfing through emasculation (Eckert et al. 2010). The question here is to asses if pollinator decline translates into higher pollen limitation for plants. The few studies that have explored the effect of pollinator decline on pollen limitation have revealed mixed conclusions. Pauw and Hawkins (2011) have produced an interesting study using herbarium specimens in the oilsecreting orchid species Pterygodium catholicum that depends on the oil-collecting bee Rediviva peringueyi (Melittidae) for its pollination. They used the pollinarium removal rates to estimate pollination activity from herbarium specimen. They established a pollination time series and showed that pollinarium removal declined in time. Though the lack of pollinarium removal does not equate to pollen limitation, this study suggests a temporal decrease in pollinator activity. Another herbarium-based study analyzed a century of pollination success 
through seed production in outcrossing Fabaceae in China (Duan et al. 2019). Overall, the data does not reveal a clear decline in seed production. Over the 109 species analyzed (4637 specimens), four out of 95 species (with at least ten samples) exhibited a significant decline. Surprisingly, nine species exhibited an increase in seed production over time. The data were however highly variable and noisy, which limits the ability to detect a clear trend. In Erythronium grandiflorum, Thomson (2010) reported results from a longitudinal survey using natural and supplementary pollination. In the 2010's paper, Thomson (2010) concluded to a pollination deterioration from 1993 to 2009 over 17 years. However, after completing the time series, Thomson (2019) concluded that this trend was not significant anymore over 26 years (from 1993 to 2018). The results are illustrated in Figure 2. This study underlines the difficulty to capture long term trends, given inherent year-to-year variation of pollination, and the need to gather data on the long run.

A recent study by Martin et al (2019) analyzed pollen limitation in France using crop yield in agrosystems. The authors classified crops according to their dependence to pollinators. It is expected that highly dependent crops will have reduced yield in poor pollination environment while such reduction is not expected in non-dependent crops. Using a 10-years dataset, the authors were able to estimate a relative pollination index at the level of French administrative department. They found that the South of France has a high values index while North of France (specifically departments around Paris) had the lowest pollination services. While the study does not directly address pollination of wild plants, it provides for the first time a pollination map at a large scale that may impact wild plants.

To date, data on the impact of pollinator decline on pollination limitation are scarce but we see from the few examples that the conclusions are not clear-cut. Several lines of interpretations can be proposed. First, it is possible that, because plant-pollinators interactions are in general imbalanced, a pollinator lost can be replaced by another one. In the peculiar case of the oil-collecting bee Rediviva peringueyi (Melittidae) and the guild of oil-secreting orchids (Pauw and Hawkins 2011) a single pollinator species interacts with the plant species and the loss of pollinator translates into a decline in pollinarium removal over time. In accordance with this interpretation, Jacquemin (2020) found that specialist plant species have declined more than generalist plant species during the 20th century in Belgium. Though is difficult to predict the future effects of pollinator decline, we may fear that the ongoing decline may produce sudden effects on plant populations (tipping point) when pollinator redundancy will become critical. Second, there may be a potential experimental problem 
when pollen limitation is inferred from seed production directly. If plants are plastic in their ability to self-fertilize ovules, a reduction in outcrossed pollen will not necessarily translate into a total seed production decrease because outcrossed seeds may be replaced by autonomous selfed seeds (Eckert et al. 2010). Ideally, self-incompatible plant would be good model to test the impact of pollinator decline on pollen limitation because they prevent the possibility of outcrossing seeds replacement by selfed seeds. We may also expect that the effect of pollinator decline on pollen limitation will be more pronounced in "simplified ecosystems" where plant-pollinator networks are simplified and the redundancy is lower. This is typically what we expect in agrosystems where pollinators are threatened by human activities (low plant diversity, pesticides, habitat fragmentation,...), in line with the large scale study of Martin et al. (2019). In accordance with this interpretation, Perrot et al. (2018) found that sunflower yield is positively correlated to the total bee abundance and bee genera diversity. Considering crops as phytometers in agrosystems, this indicates that weed pollination has likely suffered from pollinator decline.

\section{3-Can populations respond to changes in pollinator environment?}

Elements of theory. Pollination ecology has developed metrics to estimate pollen limitation in natural populations. Eckert et al. (2010) have summarized the pollination parameters and pollination manipulation to achieve them. The proportional decrease in seed production from emasculated flowers (relative to non-manipulated flowers) allow to evaluate how autonomous selfing contribute to seed production (reproductive assurance). Such techniques have been intensively used in pollination experiments as reproductive assurance is one of the key parameters in the evolution of self-fertilisation (see Lloyd 1979). Indeed, the evolution of selffertilization is basically governed by three factors. Pollen limitation (reproductive assurance) and the Fisherian cost of outcrossing select for higher selfing, while inbreeding depression selects for higher outcrossing. It is tempting to predict that pollen limitation will necessarily increase selfing but rigorous analysis of the model shows that is not necessarily the case. Evolution can drive pollination strategy towards outcrossing, even in the presence of pollen limitation. This means that evolving a fraction of unfertilized ovules can be evolutionarily stable, which provides a rationale for natural plant populations to be pollen limited (Burd 1994). However, if pollinator decline translates into higher pollen limitation, we can make the general prediction that the strength of selection towards selfing should increase.

Microevolution of reproductive systems. In plants, many traits are involved in mating system strategy. For instance, anther-stigma distance has been shown to be tightly linked to 
outcrossing rate. Floral scents and flower colours are involved in pollinator attraction defining the guild of pollinator as well the intensity of pollinator visits (Ashman and Majetic 2006). Because floral traits are under strong selection, they are $a$ priori not expected to exhibit large genetic variance. However, compiling quantitative genetics data on floral traits in a set of 41 hermaphroditic species, Ashman and Majetic (2006) found substantial heritability for floral traits associated with mating systems. For instance, they found $\mathrm{h}^{2}=0.40$ for anther-stigma separation and positioning, $h^{2}=0.45$ for corolla size, and average $h^{2}=0.20$ for nectar production. As a consequence, the conditions for mating system traits to evolve after a change in selection regime are fulfilled, in particular in the context of pollinator decline. Experimental evolution has indeed revealed that plant mating system traits can shift in a few generations. Applying various intensities of pollination, Roels and Kelly (2011) showed in Mimulus guttatus that anther-stigma distance has been reduced in the low pollination treatment after only five generations, resulting in a higher propensity to produce seeds by automous selfing. Thus, this experiment clearly shows that self-fertilisation has the potential to evolve in the context of pollinator decline. In another experimental evolution study, Gervasi and Schiestl (2017) investigated the evolution of floral traits in Brassica rapa under three different pollination treatments (hand-pollination, hoverfly and bumblebees) over nine generations. Plants pollinated by bumblebees evolved (among other traits) higher plant sizes, a more pronounced floral display and higher floral scent, while plants pollinated by hoverflies have evolved higher selfing (Figure 3). This study demonstrates that plants can rapidly adapt to a change in pollinator fauna. Interestingly the traits under selection are not necessarily floral traits in the manner of plant size. This illustrates that pollination strategy encompasses a variety of co-adapted traits that must be considered altogether.

Rapid evolution of reproductive systems in natural populations has been less studied and only a few examples have been reported. In Centaurium erythraea, Brys and Jacquemyn (2012) compared fragmented urban populations (where pollination activity is low) to non-fragmented rural populations (where pollinator activity is high). Plants from fragmented environments exhibited less herkogamy and a higher capacity for autonomous selfing than plants from nonfragmented populations (Figure 4). Assuming that urbanity is a proxy for pollinator decline, this study provides a convincing demonstration that mating systems can evolve under pollinator decline in natural populations. In agrosystems, Thomann et al. (2015) studied adaptation to pollinator decline and climate warming in the self-incompatible species Centaurea cyanus (Asteraceae) using resurrection ecology methodology. Using seeds 
sampled 18 years apart in North of France, they showed an increase in capitula size and in floral display i.e. no evidence of evolution towards selfing but rather the evolution of traits reinforcing the plant-pollinator interactions. Because the species has not evolved selfcompatibility, the authors interpreted the evolution towards higher attractiveness as a strategy to improve their fitness in an impoverished pollination environment.

Altogether, these empirical results show that rapid change in floral traits is susceptible to evolve in the face of pollinator decline. While experimental evolution has rather confirmed expectations, the direction of selection in natural populations is less clear, which suggests that the selection caused by pollinator decline is probably variable and needs to be properly characterized to make predictions about floral traits evolution.

\section{4-A little prospective for the evolution of plant communities}

Can we predict the evolution of floral traits in the face of pollinator decline? Though it would be tempting to assimilate pollinator decline to pollen limitation for plants, we see from the few empirical studies that the results are contrasted (see section 2). This question is however central and worth to be studied in the near future. In particular, we need to clarify whether pollinator community composition has changed or/and whether pollination intensity has changed. We may expect that evolutionary consequences will not be the same (see Roels and Kelly 2011 and Gervasi and Schiestl 2017 for comparison). In this respect, predicting evolutionary response to pollinator decline is not an easy task. Assuming that pollinator decline translates into pollen limitation, evolutionary models would predict that plants should evolve towards higher selfing but the few empirical results have revealed that evolutionary trajectories may differ. The evolution of larger capitula and larger floral display in the selfincompatible cornflower ( Cyanus segetum; Thomann et al. 2015) appears contradictory with other patterns of evolution such as the reduced anther stigma distance in urban populations of Centaurium erythrea resulting in higher selfing propensity demonstrated by Brys and Jacquemyn (2012). In a review, Thomann et al. (2013) analysed the literature of adaptation under low pollination and proposed that both evolution towards increased selfing or evolution toward reinforced interactions can actually occur. The lack of standing variation for some traits (e.g. no self-compatible variant in self-incompatible species) will prevent evolution, while in contrast, available standing variation will allow a rapid evolutionary trajectory. A recent study on Impatiens capensis illustrates that the two roads described by Thomann et al. 
(2013) can evolve within population. Panique and Caruso (2020) have shown that reduced pollination selected both for increased outcrossing traits in chasmogamous flowers (larger flowers) and for increased selfing through higher rate of cleistogamy.

Does mating system adaptation face to pollinator decline rescue populations? If change in pollination regimes is likely to produced evolutionary changes, whether these changes can allow plant populations to escape extinction - i.e. will provide evolutionary rescue - is worth to be studied. The evolutionary rescue is a conceptual model proposed by Gomulkiewicz and Holt (1995). It posits that, after an environmental change, evolution may help to recover a viable population that without evolution would have been doomed to extinction. This model has been tested in laboratory in microorganisms and the results show that organisms can increase their population vital rates by adapting to new conditions (temperature, $\mathrm{pH}, \ldots$ ). One can wonder if adaptation of reproductive traits in plants in the face of pollinator decline can provide such a rescue. Though it would be tempting to assert that adaption (for instance via increased selfing) would rescue populations, Cheptou (2019) showed that this is not necessarily the case in the evolution of self-fertilisation. This is due to the inherent frequencydependence selection acting on selfing traits. Analogously to the classical Hawk-dove game (Maynard Smith 1982) where the hawk strategy inevitably evolves but is deleterious once established, self-fertilization evolves through the higher replication of selfing genes (see Fisher 1941) but this does not equate to higher per-capita growth rate. In other words, the population vital rates are not maximised at the evolutionary stable selfing rate. It is thus possible, at least theoretically, that pollen limitation favours selfing causing a lower per capita growth rate i.e. allows deterministic suicide (see Figure 5). Then, if a few studies have shown that selfing can increase seed set and per capita growth rate under certain pollination circumstances (Lennartsson 2002), models for the evolution of self-fertilisation warns on the fact that conditions does not always lead to rescue. Besides, on the long run, self-fertilization is often associated to high extinction rates as it has been demonstrated in Solanaceae (Goldberg et al. 2010).

Ecosystems structure after pollinator decline. The pollination biology and plant mating system is deeply rooted in the population biology paradigm centred on the plant species, the crisis of pollinators worldwide has however recalled that the realised mating system is in most cases the results of a pollination strategy in a given pollination environment. Moreover, if pollinator decline is expected to feedback on plants (the purpose of this article), plant adaptation will feedback on pollinator through changes in pollen and nectar production. It is 
now admitted that pollinator decline has been caused by many factors, among others, pesticides and pollution i.e. factors that are external to the plant-pollinator interactions. The possibility for plants to adapt rapidly to impoverished pollination environment (as shown by experimental evolution) may be considered as a way to tone down the deleterious effects of anthropic activities. Several lines of arguments suggest that evolution may, in certain circumstances, not alleviate the effects of anthropic activities. First, the possibility of evolutionary suicide by selfing suggests that some plant populations could go extinct in communities. Second, we may naively expect that plant and pollinator populations will recover if sanitary conditions improve in the future (e.g. though the interdiction of the use of pesticides). However, if plant strategies have evolved reduced pollination interactions (e.g. selfing) because of the current pollinator crisis, we may fear that pollinators will not find enough food (pollen nectar,...) to maintain viable populations and that plant evolution will not be reversible as selfing is classically considered as a dead-end evolution (Takebayshi and Morell 2001). More than pollination strategies, the consequences of pollinator decline could be the loss of major trophic relationships in ecosystems.

\section{5- Conclusion}

The aim of this paper was to analyse if pollinator decline translates into pollen limitation for plants and eventually, how plants can adapt to such environmental change. Literature survey indicates that empirical studies are scarce and, to date, we do not have a clear picture of the effect of pollinator decline on plants. It is likely that such effects vary from ecosystems to ecosystems and that human-altered ecosystems are more affected than more natural ones. Nevertheless, we crucially lack of long-term data. On the other hand, the capacity of plants to evolve in the face of reduced pollination is now clearly established in experimental evolution. Given the current threat on pollinators, we can then conjecture that plant evolution will occur and that plant-pollinator interactions will be durably affected. 


\section{References}

Ashman, T. L. and C. J. Majetic. 2006. Genetic constraints on floral evolution: a review and evaluation of patterns. Heredity 96: 343-352.

Astegiano, J., F. Massol, M. M. Vidal, P. O. Cheptou and P. R. Guimaraes. 2015. The Robustness of Plant-Pollinator Assemblages: Linking Plant Interaction Patterns and Sensitivity to Pollinator Loss. Plos One 10(2).

Bascompte, J., P. Jordano, C. J. Melian and J. M. Olesen. 2003. The nested assembly of plantanimal mutualistic networks. Proceedings of the National Academy of Sciences of the United States of America 100(16): 9383-9387.

Baude M, Kunin WE, Boatman ND, Conyers S, Davies N, Gillespie MAK, et al. 2016. Historical nectar assessment reveals the fall and rise of floral resources in Britain. Nature. 530(7588):85-+.

Biesmeijer, J. C., S. P. M. Roberts, M. Reemer, R. Ohlemuller, M. Edwards, T. Peeters, A. P. Schaffers, S. G. Potts, R. Kleukers, C. D. Thomas, J. Settele and W. E. Kunin. 2006. Parallel declines in pollinators and insect-pollinated plants in Britain and the Netherlands. Science 313: $351-354$.

Brys, R. and H. Jacquemyn. 2012. Effects of human-mediated pollinator impoverishment on floral traits and mating patterns in a short-lived herb: an experimental approach. Functional Ecology 26(1): 189-197.

Burd, M. 199).Bateman's principle and plant reproduction: The role of pollen limitation in fruit and seed set. The Botanical Review 60(1): 83-139.

Cheptou, P. O. 2019. Does the evolution of self-fertilization rescue populations or increase the risk of extinction? Annals of botany 123(2): 337-345.

Duan, Y. W., H. B. Ren, T. Li, L. L. Wang, Z. Q. Zhang, Y. L. Tu and Y. P. Yang. 2019. A century of pollination success revealed by herbarium specimens of seed pods. New Phytologist 224(4): 1512-1517.

E-Vojtko, A., F. de Bello, W. Durka, I. Kuhn and L. Gotzenberger. 2020. The neglected importance of floral traits in trait-based plant community assembly. Journal of Vegetation Science 31(4): 529-539.

Eckert, C. G., S. Kalisz, M. A. Geber, R. Sargent, E. Elle, P. O. Cheptou, C. Goodwillie, M. O. Johnston, J. K. Kelly, D. A. Moeller, E. Porcher, R. H. Ree, M. Vallejo-Marin and A. A. Winn. 2010. Plant mating systems in a changing world. Trends in Ecology \& Evolution 25(1): 35-43.

Fisher, R. A. 1941. Average excess and average effect of a gene substitution. Annals of Eugenics. 11: 53-63.

Gervasi, D. D. L. and F. P. Schiestl. 2017. Real-time divergent evolution in plants driven by pollinators. Nature Communications $\mathbf{8}$.

Goldberg, E. E., K. J.R., R. Lande, K. Robertson, S. A. Smith and B. Igic. 2010. Species selection maintains self-incompatibility. Science 330: 493-495.

Gomulkiewicz, R. and R. D. Holt. 1995. When does the evolution by natural selection prevent extinction. Evolution 49(1): 201-207.

Goulson, D., E. Nicholls, C. Botias and E. L. Rotheray. 2015. Bee declines driven by combined stress from parasites, pesticides, and lack of flowers. Science 347(6229). Hallmann, C. A., M. Sorg, E. Jongejans, H. Siepel, N. Hofland, H. Schwan, W. Stenmans, A. Muller, H. Sumser, T. Horren, D. Goulson and H. de Kroon. 2017. More than 75 percent decline over 27 years in total flying insect biomass in protected areas. Plos One 12(10). Jacquemin, F., C. Violle, F. Munoz, G. Mahy, P. Rasmont, S. P. M. Roberts, S. Vray and M. Dufrene 2020. Loss of pollinator specialization revealed by historical opportunistic data: Insights from network-based analysis. Plos One 15(7). 
Lennartsson, T. 2002. Extinction thresholds and disrupted plant-pollinator interactions in fragmented plant populations. Ecology 83(11): 3060-3072.

Lloyd, D. G. 1979. Some reproductive factors affecting the selection of self-fertilization in plants. American Naturalist 113(1): 67-79.

Lloyd, D. G. 1992. Self and cross fertilization in plants. 2-The selection of self fertilization. International Journal of Plant Science. 153(3): 370-380.

Martin G, Fontaine C, Accatino F, Porcher E. 2019. New indices for rapid assessment of pollination services based on crop yield data: France as a case study. Ecological Indicators. 101:355-63.

Maynard Smith, J. 1982. Evolution and the theory of games, Cambrige university press. Panique, H. and C. M. Caruso. 2020. Simulated pollinator declines intensify selection on floral traits that facilitate selfing and outcrossing in Impatiens capensis. American Journal of Botany 107(1): 148-154.

Pauw, A. and J. A. Hawkins. 2011. Reconstruction of historical pollination rates reveals linked declines of pollinators and plants. Oikos 120(3): 344-349.

Perrot T, Gaba S, Roncoroni M, Gautier JL, Saintilan A, Bretagnolle V. 2019. Experimental quantification of insect pollination on sunflower yield, reconciling plant and field scale estimates. Basic and Applied Ecology. 34:75-84.

Rejou-Mechain, M. and P. O. Cheptou. 2015. High incidence of dioecy in young successional tropical forests. Journal of Ecology 103(3): 725-732.

Rhodes, C. J. 2018. Pollinator decline - an ecological calamity in the making? Science Progress 101(2): 121-160.

Roels, S. A. B. and J. K. Kelly. 2011. Rapid evolution caused by pollinator loss in Mimulus guttatus. Evolution 65(9): 2541-2552.

Takebayshi, N. and P. L. Morell. 2001. Is self-fertilization an evolutionary dead end?

Revisiting an old hypothesis with genetic theories and macroevolutionary approach. American Journal of Botany 88(7): 1143-1150.

Thomann, M., E. Imbert, C. Devaux and P. O. Cheptou. 2013. Flowering plants under global pollinator decline. Trends in Plant Science 18(7): 353-359.

Thomann, M., E. Imbert, R. C. Engstrand and P. O. Cheptou. 2015. Contemporary evolution of plant reproductive strategies under global change is revealed by stored seeds. Journal of Evolutionary Biology 28(4): 766-778.

Thomson, J. D. 2010. Flowering phenology, fruiting success and progressive deterioration of pollination in an early-flowering geophyte. Philosophical Transactions of the Royal Society B-Biological Sciences 365(1555): 3187-3199.

Thomson, J. D. 2019. Progressive deterioration of pollination service detected in a 17-year study vanishes in a 26-year study. New Phytologist 224(3): 1151-1159.

Vazquez, D. P., N. Bluthgen, L. Cagnolo and N. P. Chacoff. 2009. Uniting pattern and process in plant-animal mutualistic networks: a review. Annals of Botany 103(9): 1445-1457. 


\section{Figure legends}

Figure 1: An example of pollinator range contraction in the last decades. The range limits of the species Bombus distinguendus have drastically reduced during the last fifty years (data from the National Biodiversity Network, illustrations courtesy of Dave Goulson).

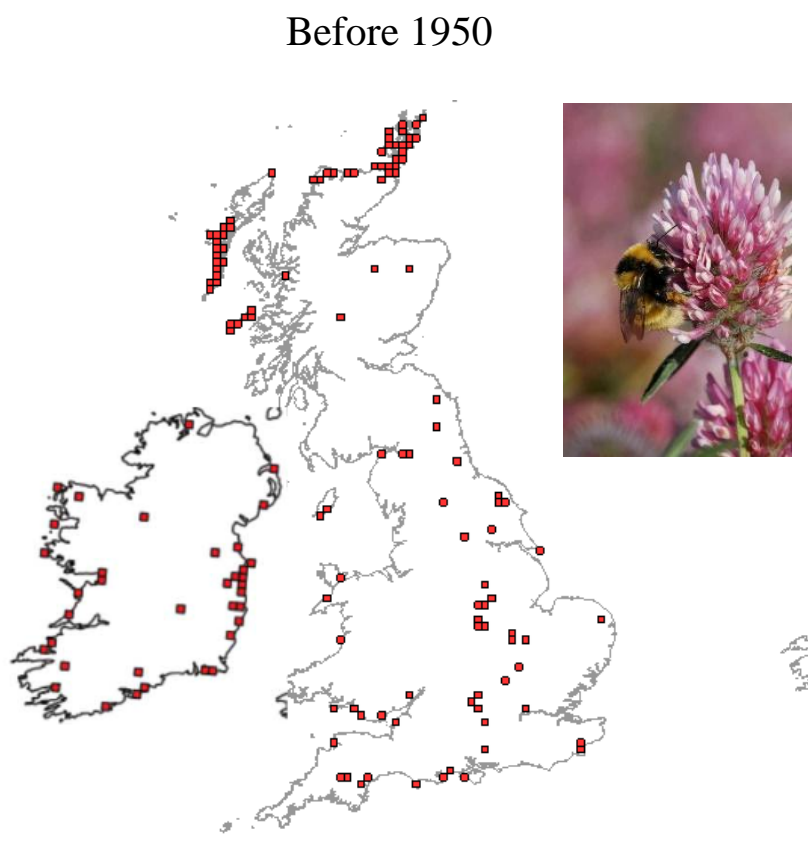

Post 2000

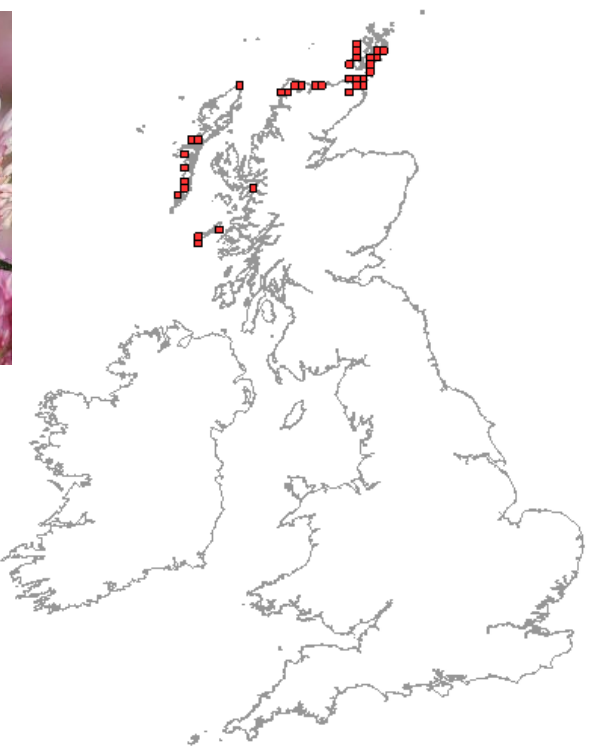


Figure 2: Estimates of pollen limitation in Erythronium grandiflorum lilies at Irwin (Colorado) from 1993 to 2018 estimated from supplemented and natural fruit production. The fruit set limitation index is defined as the fractional fruit set of supplemented plants/sum of fractional fruit sets for supplemented and control treatments (a value of 0.5 represents equal fruit production in both treatments). White dots report fruit limitation for early flowering plants, grey dots report fruit limitation for middle flowering plants and black dots report fruit limitation for late flowering plants (redrawn from Thomson 2019).

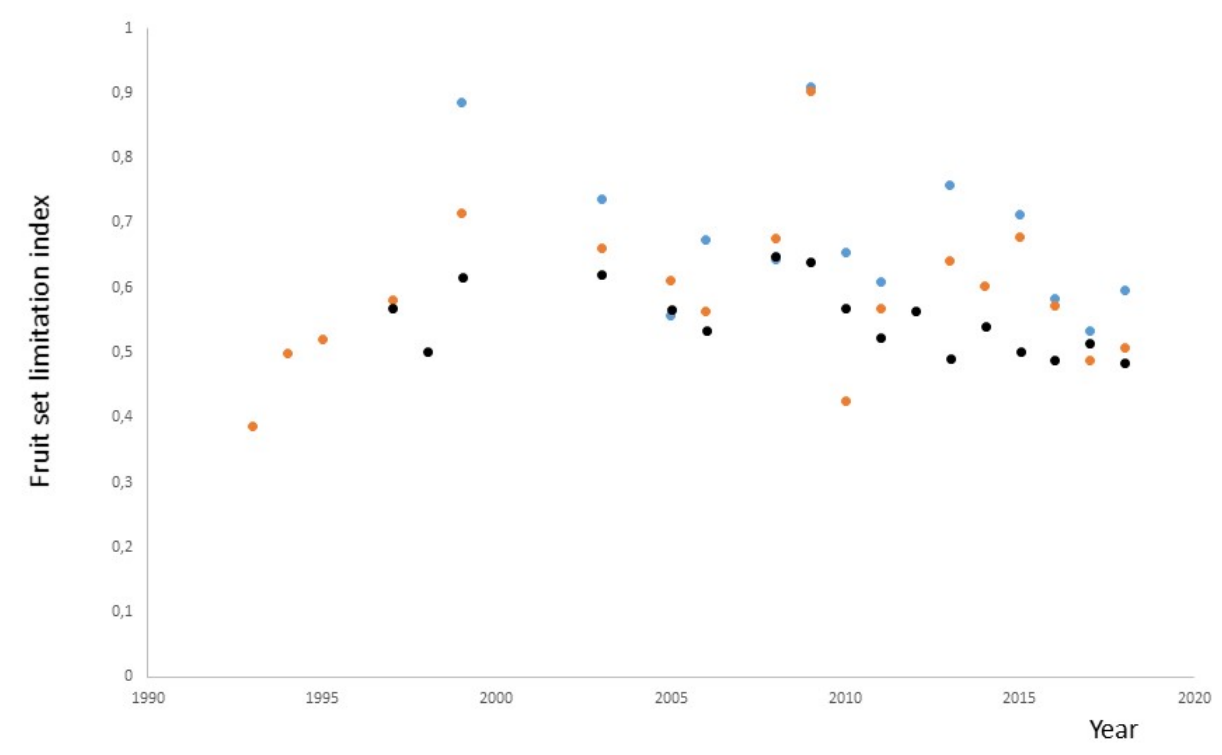


Figure 3: Experimental evolution by manipulating pollination environment with fast cycling Brassica rapa plants. Plants were submitted to (1) hand pollination, (2) hoverfly pollination, and (3) bumblebee pollination for 11 generations. The graph below reports trait shifts in the two dimensions of the linear discriminant function analysis (bumblebee: blue circles, hoverfly: green squares, control hand pollination: black triangles; filled symbols are group centroids of replicates). The results show that pollination treatments have led to significant evolutionary changes. Though not directly visible on this graph, plants having evolved under bumblebee pollination were taller, with increased ultraviolet reflection of flowers. Plants having evolved under hoverfly pollination had reduced emission of some floral volatiles, but a higher self-pollination ability (after Gervasi and Schielst 2017, illustrations courtesy of Florian Schielst).

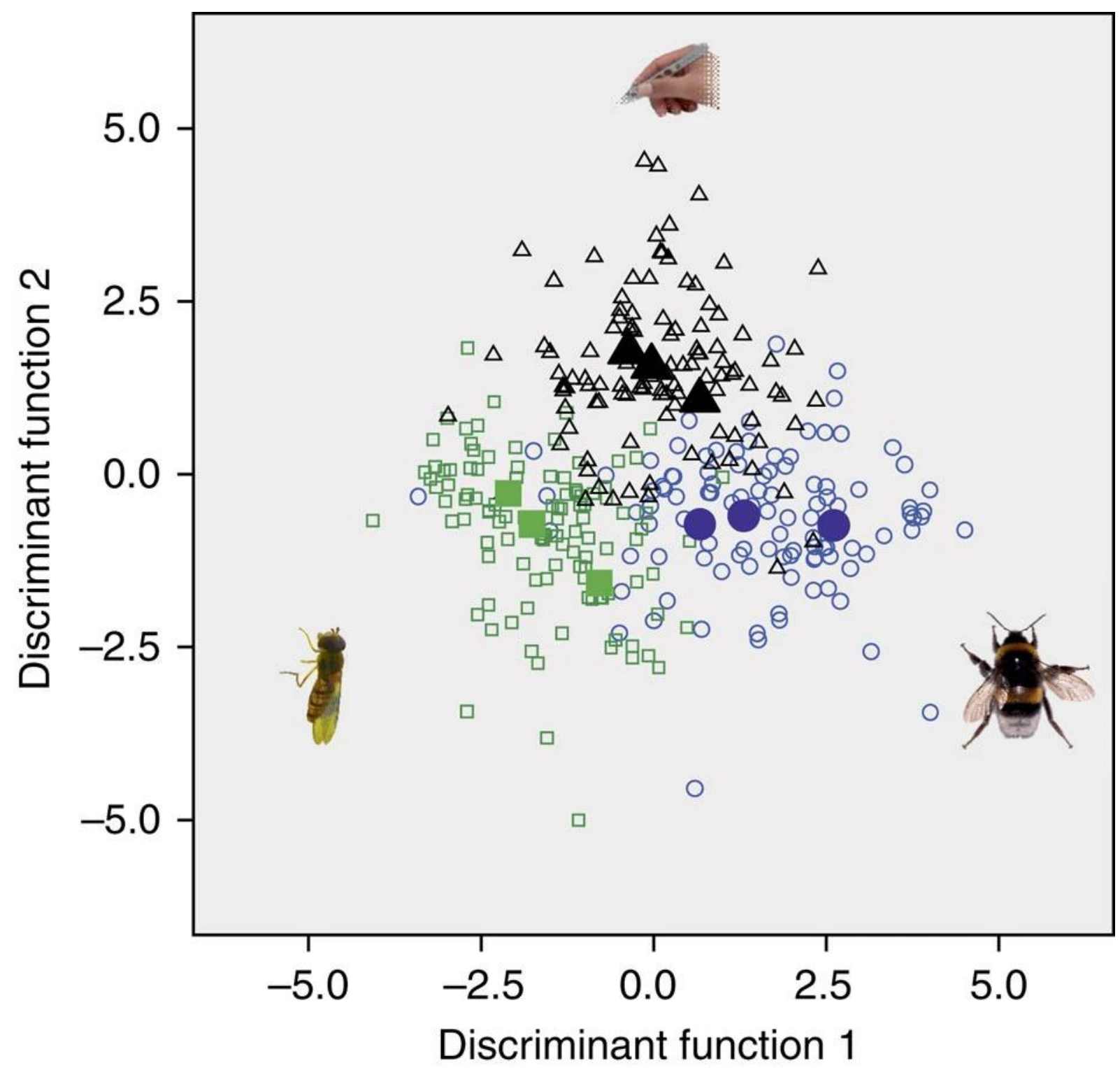


Figure 4: Reduction of anther-stigma distance in Centaurium erythreae in urban populations. On the left (a), in rural populations, the shape of the flower shows a spatial separation of the male (stamens) and female (pistil) organs favoring outcrossing, while in urban populations (on the right, b) the proximity of male and female organs favors self-fertilization (modified from Brys and Jacquemyn 2012).
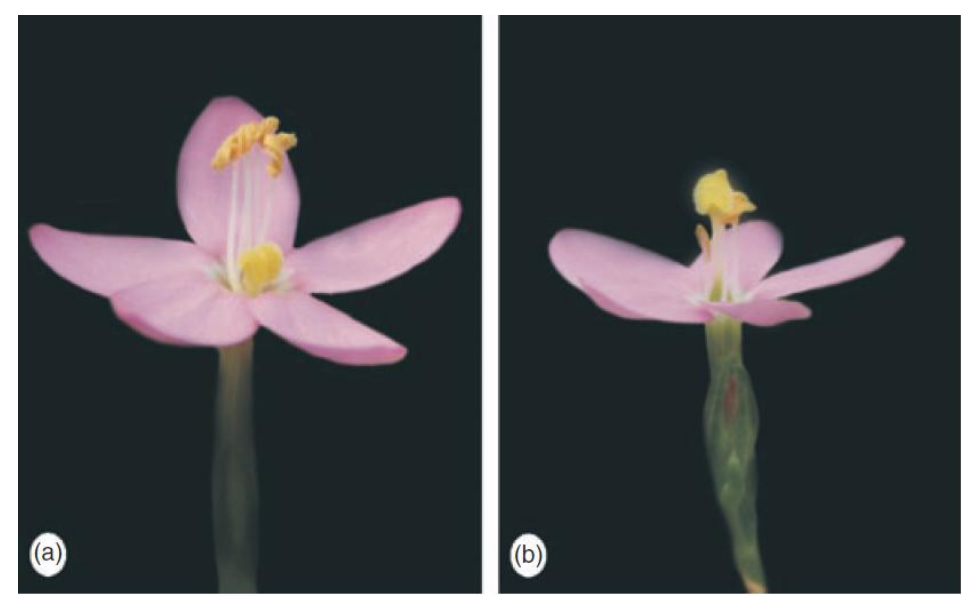
Figure 5: Evolution of self-fertilization and its consequences on demography. The evolution of self-fertilisation is based on the seminal model developed by David Lloyd (Lloyd, 1992) where evolution is driven by the cost of outcrossing, inbreeding depression $(\delta)$ and pollination rate (e). Evolution of selfing: Lloyd's model has established that higher self-fertilization (respectively higher outcrossing) evolves if $\delta<1-e / 2$ ) (respectively if $\delta>1-e / 2$ ). In the figure, the threshold for selfing/outcrossing evolution is indicated by the bold line $(\delta=1-\mathrm{e} / 2)$. Demography of selfing populations: Cheptou (2019) established that the per capita growth rate increases (respectively decreases) with selfing if $\delta<1-e$ (respectively if $\delta>1-e)$. In the figure, the threshold is indicated by the bold line $(\delta=1-\mathrm{e} / 2)$ above which the per capita in higher and below which, the per capita is lower.

Conclusion: Because the evolution of selfing increases or decreases the per capita growth rate, the evolution of selfing can either provide evolutionary rescue (below the thin line) or evolutionary suicide (above the thin line) (from Cheptou, 2019).

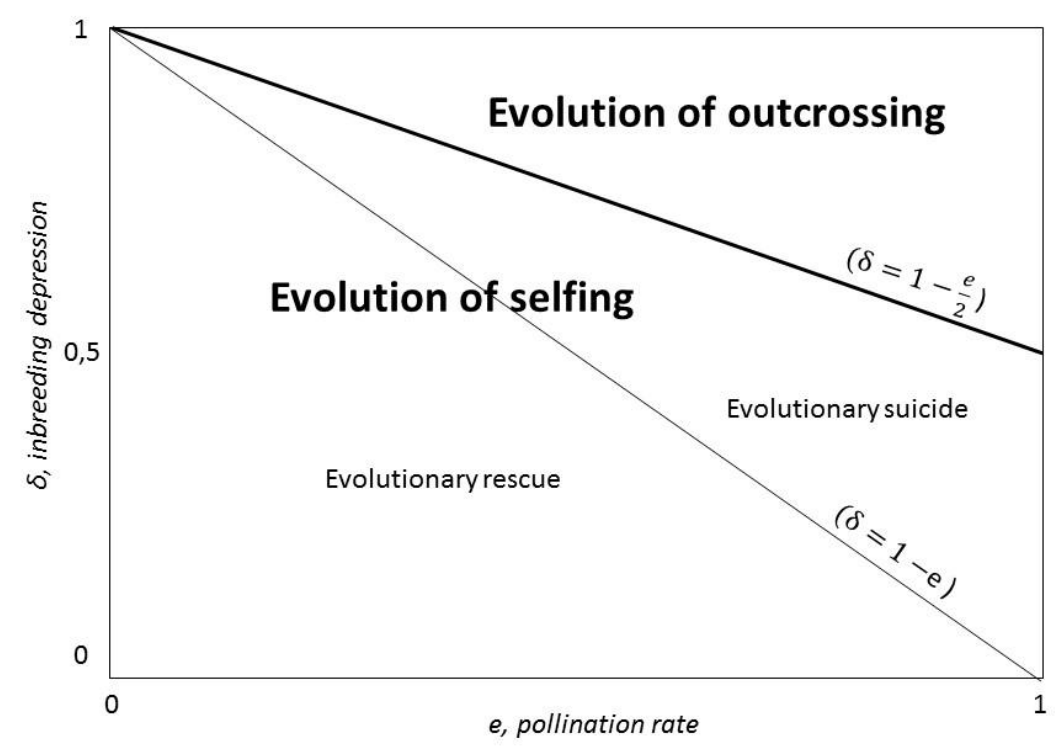

